\title{
Exposición a contenidos de uso de alcohol en Facebook y consumo de alcohol en adolescentes.
}

\author{
Edna Idalia Paulina Navarro-Oliva1,a, Paola Jazmín De Anda Robledo, \\ Mario Enrique Gámez Medina ${ }^{1, c}$, Josefina Saraí Candía-Arredondo ${ }^{1, a}$, \\ Francisco Rafael Guzmán Facundo ${ }^{1, c}$
}

\section{RESUMEN}

El consumo de alcohol en adolescentes ha aumentado en los últimos años, nuevas plataformas pueden favorecer el consumo de alcohol tales como las redes sociales (Facebook). Objetivos: describir la relación entre la exposición de contenidos de consumo de alcohol en Facebook y el consumo de alcohol. Material y Métodos: diseño descriptivo correlacional. La población fueron adolescentes de Bachillerato del área metropolitana de México,76 adolescentes fueron encuestados. Se utilizó una cédula de datos personales, el cuestionario de Identificación de Trastornos por Uso del Alcohol (AUDIT) y Libro de Códigos para Facebook (LCCAF).Resultados: el 61,8\% mostraron contenidos relacionados con el consumo de alcohol en Facebook. 65,8\% refirió consumo de alcohol en el último año y 38,2\% en el último mes. Se mostró diferencia en las puntuaciones del AUDIT por exposición de contenidos de alcohol en Facebook $(\chi 2=17,66, p<0,001)$, destacando puntuaciones mayores de AUDIT en los adolescentes con exposición en Facebook de problemas de consumo de alcohol $(15,4)$ y consumo de alcohol visualizado en Facebook $(5,3)$ comparado con aquellos que no tuvieron visualizaciones de consumo de alcohol $(3,4)$. Finalmente se mostró una relación positiva y significativa del número de exposiciones de alcohol en Facebook (fotografías, comentarios y videos) con la puntuación del AUDIT ( $r s=0,517, \mathrm{p}<0,001)$. Conclusiones: la exposición de contenidos de consumo de alcohol en Facebook se relaciona con el consumo de alcohol de los adolescentes. Es importante considerar las redes sociales como una variable importante para ser incluida en futuras intervenciones en prevención del consumo de alcohol.

PALABRAS CLAVE:consumo de alcohol, red social, adolescente, México.

\section{Exposure to alcohol use contents on Facebook and alcohol consumption in teenagers.}

\section{SUMMARY}

Alcohol use among adolescents has increased in recent years, new platforms may promote alcohol consumption such as social networks (Facebook).Objectives:To describe the relationship between exposure to alcohol content on Facebook and consumption of alcohol. Material and Methods: A descriptive correlational design. The teens were high school population of the metropolitan area of Mexico. 76 adolescents were surveyed. A Data Questionnaire Identification of Alcohol Use Disorders Test (AUDIT) and Codebook for Facebook (LCCAF) was used. Results: $61.8 \%$ showed associated with alcohol consumption in Facebook content. 65.8\% reported drinking alcohol in the last year and $38.2 \%$ last month. Showed difference in exposure AUDIT scores alcohol content Facebook $\left(\chi^{2}=\right.$ 17.66, $\mathrm{p}<0.001$ ), highlighting over AUDIT scores in adolescents with Facebook exposure of alcohol problems

Facultad de Enfermería, Universidad Autónoma de Nuevo León; Monterrey, Nuevo León, Mexico

Estudiante del Programa de Doctorado en Ciencias de Enfermería

Maestra en Ciencias de Enfermería

Doctor en Ciencias de Nefermería 
(15.4) and alcohol consumption displayed on Facebook (5.3) compared to those who did not have views of alcohol (3.4). Finally a positive and significant relationship of alcohol number of exposures on Facebook (photos, comments and videos) with AUDIT score $(\mathrm{rs}=0.517, \mathrm{p}<0.001)$ showed. Conclusion: Exposure of alcohol content on Facebook is related to alcohol consumption among adolescents. It is important to consider social networks as an important tool to be included in future interventions in prevention of alcohol consumption variable.

KEY WORDS:Alcohol consumption, social networking, adolescent, México.

\section{INTRODUCCIÓN}

El consumo de alcohol es uno de los principales problemas que afecta a diferentes poblaciones a nivel mundial por cual ya no sólo se considera como un problema de salud pública sino un problema que va más allá al considerar las consecuencias que este genera a los individuos. Una de las poblaciones que se ha situado con mayor riesgo para experimentar daños por su consumo son los adolescentes, de acuerdo a la condición de vulnerabilidad en el entorno social donde se desenvuelven. Los adolescentes se encuentran en búsqueda de su identidad, de nuevas sensaciones yexperiencias, además inician a tomar sus propias decisiones, las cuales pueden convertirse en un riesgo como es el consumo exploratorio de sustancia, ya que los adolescentes se encuentran una etapa de transición de la dependencia hacia la independencia (1-3).

Los reportes de las últimas encuestas realizadas en México indican un incremento significativo en las prevalencia de consumo de alcohol. De tal manera que el consumo en el último año pasó del $25,7 \%$ al $30,0 \%$, en el último mes del $7,1 \%$ al $14,5 \%$, sin embargo un dato que es preocupante para el sector salud son los incrementos en el índice de dependencia de alcohol que registró un incremento del $2,1 \%$ al $4,1 \%$. El inicio precoz de consumo de alcohol incrementa el riesgo de desarrollar un alto consumo o dependencia al alcohol, lo cual puede originar graves consecuencias en la salud de los adolescentes o prolongarse estos patrones de consumo a la adultez (4-6).

De acuerdo a la literatura existente se muestran los múltiples factores que influyen en el adolescente para el inicio del consumo de alcohol, dentro de estos factores uno de los más importantes son los factores sociales, es ya conocido que los medios de comunicaciónhan evolucionado y muestran una mayor cobertura de la población. En este sentido uno de los medios de comunicación más utilizado entre los adolescentes son las redes sociales como el Facebook, los cuales favorecen las relaciones sociales y la capacidad de compartir rápidamente información. Facebook ofrece una manera más fácil de compartir información, experiencias y momentos (por ejemplo, fotografías,imágenes, videos, mensajes, invitaciones, etc.,) con amigos, familiares y desconocidos. Actualmente se reporta más de 1320 millones de usuarios de Facebook activos a nivel mundial $(7,8)$.

Diversos estudios recientes realizados en Estados Unidos muestran que el uso de Facebook se ha identificado como un posible factor o estímulo para el inicio o incremento del consumo de alcohol en adolescentes, ya que estudios han evidenciado que cerca del $56 \%$ al $90 \%$ de los perfiles en Facebook de adolescentes muestran contenidos que hacen referencia al consumo de alcohol (9-14).

Es importante mencionar que en México no se ha encontrado evidencia científica sobre la exposición de contenidos de alcohol en Facebook y la relación con el consumo de alcohol en adolescentes, por lo cual se considera necesario explorar los factores sociales del consumo de alcohol en adolescentes a partir del uso de Facebook y que los resultados arrojados por esta investigación sirvan de base para el diseño de intervenciones de enfermería en la prevención de consumo de alcohol.

El objetivo del presente estudio fue explorar la relación entre la exposición de contenidos de uso de alcohol en Facebook y el consumo de alcohol en adolescentes.

\section{MATERIAL Y MÉTODOS}

El diseño del estudio fue descriptivo correlacional ya que se describió la relación de la exposición de consumo de alcohol en Facebook y el consumo de alcohol en adolescentes (15). La población se conformó por 3019 adolescentes de ambos sexos, inscritos en Colegios de Educación Profesional Técnica del Municipio de San Nicolás de los Garza, Nuevo León. El muestreo que se utilizó fue aleatorio estratificado por plantel y semestre con asignación proporcional al estrato. La muestra fue conformada por 466 adolescentes la cual fue calculada a través del paquete estadístico QueryAdvisor V 4.0 (16). 
No obstante para este artículo solo se presentan los resultados de los adolescentes que repondieron a la invitación por facebook.

Fue utilizada una Cédula de Datos Personales y Prevalencias del Consumo de Alcohol (CDP y PCA), el Cuestionario de Identificación de Trastornos por Uso del Alcohol (AUDIT) validado en población mexicana, consta de 10 reactivos que examinan el consumo de alcohol durante los últimos doce meses y sus consecuencias. Los reactivos del 1 al 3 determinan la cantidad y frecuencia del consumo de alcohol, los reactivos del 4 al 6 evalúan la posibilidad de que exista dependencia al alcohol y los reactivos 7, 8, 9 y 10 examinan el consumo dañino. Cada pregunta cuenta con una serie de respuestas a elegir y cada respuesta tiene una escala de puntuación de 0 a 4 , en general la puntuación igual o mayor a 1 en la pregunta 2 o la pregunta 3 indica un consumo de riesgo, así mismo una puntuación por encima de 0 en las preguntas de la 4 a la 6 implica la presencia o el inicio de una dependencia de alcohol y por último los puntos obtenidos en las preguntas de la 7 a la 10 indican que ya se están experimentando daños relacionados con el alcohol. El cuestionario ha sido aplicado en adolescentes del estado de Nuevo León, donde se ha obtenido un Alfa de Cronbach de 0,82 $(17,18)$.

Así mismo se utilizó el libro de códigos para contenidos de alcohol en Facebook (LCCAF), el cual es utilizado para realizar una revisón de la página personal de Facebook en busca de contenidos que hagan referencia al consumo de alcohol, este incluye la descripción mecanografiada de cualquier contenido en imagen o el texto en sus páginas de Facebook. La forma en la cual se ha utilizado este libro de codigos es mediante la observacion de varios investigadores o visualizadores de la pantalla y se establecen tres posibles respuestas las cuales soon: no visualizado, donde la página del joven no hace ninguna referencia al consumo de alcohol; alcohol visualizado, en esta se hace referencia a uno o más contenidos del consumo de alcohol, pero sin referencias de intoxicación o problemas con el alcohol, como por ejemplo se evalúa el contenido en fotografías o textos personales en las que el propietario del perfil estaba bebiendo de una botella de cerveza o referencias de texto que describen el consumo de alcohol.

Y la tercera opción es la intoxicación o problemas con el alcohol visualizado, en este se observa una o más referencias a cualquier intoxicación o comportamiento problemático relacionados con el alcohol, de acuerdo a los autores del libro de códigos se propone la utilización de la técnica CRAFFT la cual ha sido utilizada y validad en adolescentes. Incluye los criterios de manejar o ir en un vehículo bajo los efectos del alcohol (Car, coche), beber para relajarse (Relax, relajante), beber alcohol solo (Alone, solo, olvidándose de lo que uno hizo, mientras se bebía o perder el conocimiento (Forget, olvida), tener amigos o familia que le piden reducir el consumo de alcohol (Friends/Family, amigos / familia ), o meterse en problemas relacionados con el consumo de alcohol como ser arrestado (Trouble, problemas), con el fin de buscar cualquiera de estas complicaciones en su página personal $(11,14,19)$.

Es importante mencionar que esta técnica de recolección de datos se ha evaluado su confiabilidad mediante la utilización del estadístico Kappa de Cohen la identificación de las referencias al uso de alcohol fue de 0,82 , lo cual indica una casi perfecta fuerza de concordancia por parte de los visualizadores al cumplir con lo estipulado en el libro de códigos (11).

Para el procedimiento de recolección de datos se contó con la aprobación del Comité de Investigación y el Comité de Ética Investigación de la Facultad de Enfermería de la Universidad Autónoma de Nuevo León, además se solicitó por escrito la autorización para la realización del estudio a los directivos dos Colegios de Educación Profesional Técnica; posteriormente se solicitó la lista de grupos de las instituciones y se seleccionaron de forma aleatoria, una vez seleccionados los grupos, se solicitó la autorización para acudir a las aulas, en los horarios señalados por las autoridades, para interferir lo menos posible con las actividades académicas y se aplicó los instrumentos. Posteriormente se realizó un perfil de Facebook con el nombre del proyecto para realizar la invitación a los participantes y facilitar la exploración y la evaluación de los contenidos relacionados con el consumo de alcohol en los perfiles de los estudiantes, se hizo una evaluación retrospectiva de los últimos seis meses de los contenidos relacionados con el consumo de alcohol en los perfiles de los participantes que respondieron a la invitación.

Los datos fueron procesados en el programa estadístico Statistical Packageforthe Social Sciences (SPSS) para Windows V 22.0.Se utilizó estadística descriptiva para obtener frecuencias, proporciones, medidas de tendencia central (medias y medianas) y de variabilidad (desviación estándar) y estadística inferencial no parametrica para dar respuesta a los objetivos. La consistencia interna de los instrumentos se determinó a través del coeficiente de Confiabilidad Alfa de Cronbach y el Kappa de Cohen (LCCAF). 


\section{RESULTADOS}

Respecto algunas características sociodemográficas de la muestra se destaca un promedio de edad de 16,93 años $(D E=1,01)$, con una media de años de escolaridad de 11,51 años $(D E=1,39)$, el $50 \%$ de los adolescentes son mujeres, la mayoría solteros $(98 \%)$ y el $21 \%$ mencionó estudiar y trabajar. En base a la descripción de uso de Facebook, el promedio de los días de uso de Facebook por semana fue de 5,9 $(D E=1,82)$, respecto a las horas de uso de Facebook por día se observa una media de $5,7(D E=4,47)$ y en relación a los días de uso en los últimos 30 días de 22,14 $(D E=10,0)$. Los dispositivos más utilizados para acceder a Facebook, fue por medio de celulares $(78,9 \%)$, seguido de computadoras $(7,1 \%)$.

En la tabla 1, se observan los resultados de la prueba Chi Cuadrado para la exposición de contenidos sobre el consumo de alcohol en Facebook por sexo, los datos muestran que no existen diferencias significativas entre hombres y mujeres. De acuerdo a lo descrito en la tabla 2 se muestra que no existen diferencias de consumo de alcohol entre hombres y mujeres. Se destaca una prevalencia de $65,8 \%$ de consumo de alcohol en el último año en los adolescentes. En la tabla 3 muestra el contraste de tendencia central de la puntuación total del AUDIT entre la exposición de consumo de alcohol en Facebook (no visualizado, consumo visualizado, problemas de consumo visualizado) por la Prueba Kruskall- Wallis.

Los resultados muestran diferencias significativas destacando mayores puntuaciones de consumo de alcohol (AUDIT) en los adolescentes que muestran exposición de problemas de consumo de alcohol en Facebook, comparado con aquellos que solo muestran consumo visualizado y que no muestran visualizaciones de contenidos de alcohol. La tabla 4, muestra que el número

Tabla 1.Exposición de contenidos de alcohol en Facebook por sexo.

\begin{tabular}{lccccccc}
\hline \multicolumn{1}{c}{$\begin{array}{c}\text { Exposición de } \\
\text { contenido de alcohol } \\
\text { en Facebook }\end{array}$} & \multicolumn{2}{c}{ Mujeres } & \multicolumn{2}{c}{ Hombres } & \multicolumn{2}{c}{ Total } \\
\hline $\begin{array}{l}\text { Alcohol no } \\
\text { visualizado }\end{array}$ & 15 & 39,5 & 14 & 36,8 & 19 & 38,2 \\
$\begin{array}{l}\text { Alcohol visualizado } \\
\begin{array}{l}\text { Problemas de } \\
\text { consumo de alcohol } \\
\text { visualizado }\end{array}\end{array}$ & 21 & 55,3 & 19 & 50,0 & 40 & 52,6 \\
\hline Nota: $\chi^{2}=1,42, \mathrm{p}=0,492$ & $\mathrm{n}=76$ & 5,2 & 5 & 13,2 & 7 & 9,2 \\
\hline
\end{tabular}

Tabla 2. Prevalencia de consumo de alcohol alguna vez en la vida, en último año y en el último mes y últimos siete días por sexo.

\begin{tabular}{|c|c|c|c|c|c|c|c|c|}
\hline \multirow{2}{*}{$\begin{array}{l}\text { Prevalencia } \\
\text { de } \\
\text { Consumo } \\
\text { de alcohol }\end{array}$} & \multicolumn{2}{|c|}{ Hombres } & \multicolumn{2}{|c|}{ Mujeres } & \multicolumn{2}{|c|}{ Total } & \multicolumn{2}{|c|}{$95 \%$ IC } \\
\hline & $\mathbf{F}$ & $\%$ & $\mathbf{F}$ & $\%$ & $\mathbf{F}$ & $\%$ & LI & LS \\
\hline $\begin{array}{l}\text { Alguna vez } \\
\text { en la vida* }\end{array}$ & 32 & 84,2 & 30 & 78,9 & 62 & 81,6 & 72 & 90 \\
\hline $\begin{array}{l}\text { En el último } \\
\text { año }\end{array}$ & 25 & 65,8 & 25 & 65,8 & 50 & 65,8 & 54 & 76 \\
\hline $\begin{array}{l}\text { En el último } \\
\text { mes** }\end{array}$ & 14 & 36,8 & 15 & 39,5 & 29 & 38,2 & 26 & 49 \\
\hline $\begin{array}{l}\text { En últimos } 7 \\
\text { días**** }\end{array}$ & 9 & 23,7 & 7 & 18,4 & 16 & 21,1 & 11 & 30 \\
\hline $\begin{array}{l}\text { Nota: } \mathrm{IC}=\text { Interv } \\
\text { superior } \\
\mathrm{n}=76 \\
* \chi^{2}=0,350, \mathrm{p}=0, \\
* * * * \chi^{2}=0,317,\end{array}$ & , & $x^{2}=$ & 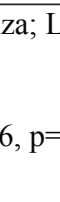 & 813 & & 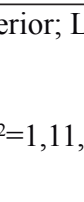 & & \\
\hline
\end{tabular}

Tabla 3. Contraste de tendencia central de la puntuación total del AUDIT entre la exposición de consumo de alcohol en Facebook (no visualizado, consumo visualizado, problemas de consumo visualizado) por la Prueba Kruskall- Wallis.

\begin{tabular}{cccccccc}
\hline $\begin{array}{c}\text { Exposición de } \\
\text { consumo de } \\
\text { alcohol por } \\
\text { Facebook }\end{array}$ & $\mathbf{N}$ & $\%$ & Mdn & DE & $\chi^{2}$ & gl $\begin{array}{c}\text { Valor } \\
\text { de p }\end{array}$ \\
\hline $\begin{array}{c}\text { No visualizado } \\
\text { Consumo }\end{array}$ & 12 & 3,33 & 3,00 & 2,38 & & & \\
$\begin{array}{c}\text { Visualizado } \\
\text { Problemas de } \\
\text { consumo }\end{array}$ & 31 & 5,41 & 5,00 & 3,96 & 17,66 & 2 & 0,001 \\
$\quad$ Visualizado & 7 & 15,85 & 15,00 & 5,08 & & & \\
\hline $\mathrm{n}=76$
\end{tabular}
$\overline{\mathrm{n}}=76$

de exposición a contenidos que involucran alcohol se relacionó positiva y significativamente con el consumo del alcohol $(r=0,517, p<0,001)$, con la frecuencia $(r=0,317$, $p<0,05)$, dependencia $(r=0,42, p<0,05)$ y consumo prejudicial $(r=0,456, \quad p<0,001)$, lo que significa que a mayor número de exposiciones de contenidos tales como fotografías, leyendas, anuncios que involucran el consumo de alcohol, mayor es la frecuencia de consumo, mayores los síntomas de dependencia y mayor es el consumo prejudicial. En la figura 1 se muestra la representación gráfica de esta relación. 
Tabla 4.Coeficiente de Correlación de Spearman para número exposiciones con contenidos de consumo de alcohol y puntuaciones de AUDIT.

\section{Variable AUDIT Frecuencia Dependencia Perjudicial}

$\begin{aligned} & \text { Numero de exposiciones de } \\ & \text { contenidos de consumo de } \\ & \text { alcohol en Facebook }\end{aligned}$
Nota: $* * 517 * * \quad 0,371 * 0,001 .{ }^{*} \mathrm{p}<0,05$

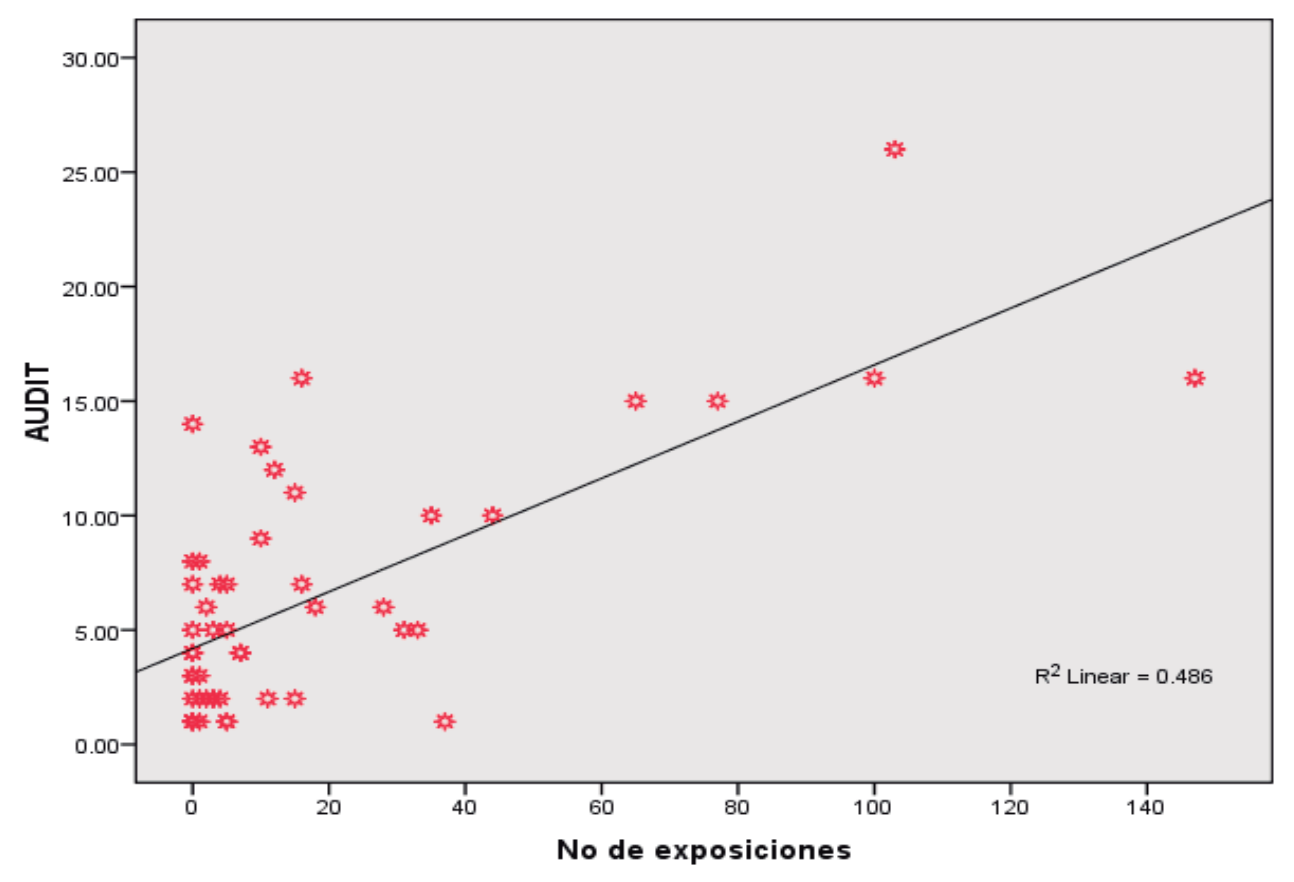

Figura 1. Representación gráfica de la relación entre el número de exposiciones de consumo de alcohol en Facebook y el consumo de alcohol.

\section{DISCUSIÓN}

Este estudio permitió explorar la relación entre la exposición del consumo de alcohol en Facebook con el consumo de alcohol en adolescentes. Respecto a la exposición de uso de Facebook resulto que los adolescentes utilizan la red social cerca de 6 días a la semana con un promedio de 5,7 horas al día, estos resultados difieren con otros estudios realizados principalmente en Estados Unidos que marcan un promedio de uso diario de entre 30 y 90 minutos al día (20-23).Esto puede deberse al constante incremento del uso de nuevas tecnologías en los adolescentes para mantener vínculos con sus amistades de una forma más rápida.

En cuanto a la exposición a contenidos de alcohol en Facebook, los jóvenes reciben en promedio 12 exposiciones semestralmente incluyendo fotografías, mensajes, publicidad e invitaciones a fiestas que involucran alcohol. Estos resultados son semejantes a lo reportado en la literatura reciente, esto podría tener diversas explicaciones, una de ellas es que en los últimos años el aumento de usuarios a esta red social electrónica se vuelve atractiva para las empresas (en este caso las cerveceras) invertir en publicidad y hacer aparecer sus anuncios en los muros de los usuarios de Facebook $(24,25)$. Otra explicación podría ser la intensidad de uso de Facebook en este estudio (días y horas de uso) que fue mayor que en los estudios encontrados lo que conlleva a una mayor exposición y probabilidad de ver anuncios y publicaciones en el muro relacionados con el alcohol (26).

Otro hallazgo es lo relacionado al número de amigos en Facebook, donde el adolescente conoce en persona 
aproximadamente a la mitad de sus contactos en Facebook y el dispositivo que con mayor frecuencia utilizan para conectarse a Facebook es el celular con más del 70\% de los casos, en parte al fácil acceso de las nuevas tecnologías (Smartphones) a la red social electrónica (27). Además el consumo de alcohol se relacionó con el número de exposiciones de Facebook relacionadas con el alcohol, esto concuerda con los resultados de diferentes estudios $(14,24,27)$. Con base a la teoría del aprendizaje social, la exposición al consumo de alcohol en los medios de comunicación puede influir en el comportamiento a través de la promoción de actitudes positivas e intenciones hacia el comportamiento que se muestra (28-31), así mismo, la exposición a los contenidos relacionados con el alcohol en Facebook pueden crear o proyectar en el adolescente una imagen "positiva" basada en la percepción de la normatividad social del consumo de alcohol en este sentido se ha demostrado que las personas tratan de hacer autorepresentaciones positivas en Facebook, usualmente al publicar fotografías o mensajes en el muro que son socialmente deseables como eventos positivos o momentos felices. En la vida real el consumo de alcohol suele estar implicado en esos momentos felices, especialmente entre jóvenes (fiestas, reuniones, festejos). La representación positiva del consumo de alcohol en las redes sociales electrónicas como Facebook puede en parte ser explicada por la exposición de los momentos felices (13).

\section{CONCLUSIONES}

Se mostró una alta relación entre el número de exposiciones con contenidos de consumo de alcohol y el consumo de alcohol en adolescentes. Es decir a mayores exposiciones con contenidos de consumo de alcohol mayor es el consumo de alcohol en adolescentes.Por otra parte es importante mostrar interés por explorar las problemáticas de los adolescentes desde la perspectiva de los mismos adolescentes o jóvenes. Así mismo se recomienda el abordar variables que puedan estar favoreciendo la influencia del uso de Facebook hacia el consumo de alcohol, una de ellas podría ser la persuasión que ejerce este medio de comunicación para que los adolescentes puedan sentir inducidos hacia el consumo de alcohol.

El principal aporte del presente estudio es mostrar una realidad existente aunque es subestimada al no considerar que la exposición de contenidos de consumo de alcohol podría afectar a la población. Se recomienda comenzar una iniciativa a través de las redes sociales para la prevención del uso de alcohol en la adolescencia mostrándoles a los mismos adolescentes como el Facebook como red social puede influir en conductas de riesgo como es el abuso en el consumo del alcohol. Finalmente es importante considerar el Facebook o demás redes sociales como una variable importante para ser incluida en futuras intervenciones en prevención del consumo de alcohol.

\section{REFERENCIAS BIBLIOGRÁFÍCAS}

1. Alcívar C, Calderón J, Jácome N. ¿El uso de las drogas afecta a la sociedad o "contribuye al turismo"? Y sus riesgos en los jóvenes y adolescentes. Revista Contribuciones a las Ciencias Sociales. 2015;1: 27. (Citado el 20 Enero 2015). Disponible en: http://www.eumed.net/rev/ cccss/2015/01/drogas.html

2. National Institute on Alcohol Abuse and Alcoholism. Statistical snapshot of college drinking. Washington: National Institute on Alcohol Abuse and Alcoholism;2010. (Citado el 27 Abril 2012). Disponible en: http://www.niaaa. nih.gov...StatisticalSnapshotCollegeDrinking.htm

3. Spear L. Adolescent Neurodevelopment. Journal of Adolescent Health. 2013; 52: S7-S13.

4. Consejo Nacional Contra las Adicciones, Secretaría de Salud. Encuesta Nacional de Adicciones Alcohol. Col. Juárez Deleg. Cuauhtémoc D.F.:Consejo Nacional Contra las Adicciones;2011.

5. Velázquez A, Arellanez H, Martínez, G. Asertividad y Consumo de Drogas en Estudiantes Mexicanos. Acta Colombiana de Psicología. 2012; 15 (1): 131-141.

6. Villatoro V, Gaytán F, Moreno L, Gutiérrez L, Oliva R, Bretón C. Tendencias del uso de drogas en la Ciudad de México: Encuesta de Estudiantes del 2009. Salud Mental. 2011; 34(2): 81-94.

7. Boyd D, Ellison N. Social network sites: definition, history and scholarship. Journal of ComputerMediatedCommunication. 2007; 13: 210-230.

8. Goodwill Community Foundation. Social media: Facebook 101. North Carolina: Goodwill Community Foundation; 2014.

9. Egan K, Moreno M. Alcohol references on undergraduate males Facebook profiles. Am J Mens Health. 2011; 5(5): 413-420.

10. Glassman T. Implications for collage students posting picture of themselves drinking alcohol on Facebook. Journal of Alcohol \& Drug education. 2012; 56 (1): 38-58.

11. Moreno M, Christakis D, Egan K, Brockman L, Becker T. Associations between displayed alcohol references on Facebook and problem drinking among college students. Arch Pediatr Adolesc Med. 2012; 166 (2): 157-163.

12. Moreno M, Grant A, Kacvinsky L, Egan K., Fleming M. Collage students'alcohol displays on Facebook: intervention considerations. Journal of American Collage health. 2012; 60 (5): 388-394.

13. Beullens K, Schepers A. Display of alcohol use on Facebook: a content analysis. Cyberpsychology, Behavior, and Social Networking. 2013; 16 (7): 497-503.

14. Fournier AK, Clarke SW: Do college students use Facebook 
to communicate about alcohol? An analysis of student profile pages. Journal of Psychosocial Research on Cyberspace.2011; 5: 2-5.

15. Burns N, Grove S. The practice of nursing research: Conduct, critique and utilization. 5 ed. Missouri: Elsevier Saunders; 2005.

16. Elashoff J. nQuery Advisor (Versión 7.0) [Software de computación]. Los Angeles, CA: Statistical Solutions LTD; 2007.

17. De la Fuente J, Kershenobich D. El alcoholismo como problema médico. Revista Facultad de Medicina UNAM. 1992; 35(2): 47-51.

18. Gómez M, Gómez H, Morales R, Pérez R. Uso del AUDIT y el DAST-10 para la identificación de abuso de sustancias psicoactivas y alcohol en adolescentes. Revista Colombiana de Psicología. 2009; 18(1): 9-17.

19. Moreno, M, Briner L, Williams A, Brockman L, Walker L, Christakis D. A content analysis of displayed alcohol references on a Social Networking Web Site. The Journal of Adolescent Health. 2010; 47(2): 168-175.

20. Ellison N, Steinfield C, Lampe C. The benefits of Facebook "friends:" social capital and collage students' use of online social network sites. Journal of Computer-Mediated Communication. 2007; 12(4): 1143-1168.

21. Forest A, Wood J. When social networking is not working: individuals with low self-esteem recognize but do not reap the benefits of self-disclosure on Facebok. Psycological Science. 2012; 23(3): 295-302.

22. Skues J, Williams B, Wise L. The effects of personality traits, self-esteem, loneliness, and narcissism on Facebook use among university students. Computers in Human Behavior. 2012; 28: 2414-2419.

23. Pettjohn T, LaPiene K, Pettjohn T, Horting A. Relationships between Facebook intensity, friendship contingent self-esteem, and personality in U.S. collage students. Cyberpsychology. 2012; 6(1). DOI: 10.5817/CP2012-1-2.

24. Crow B. Does Facebook impact college students' alcohol consumption? A dissertation submitted in partial fulfillment of the requirements for the doctor of philosophy. Chicago Illinois: University Carbondale; 2013.
25. Griffiths R, Casswell S. Intoxigenic digital spaces? Youth, social networking sites and alcohol marketing. Drug and Alcohol Review. 2010; 29: 525-530.

26. Epstein J. Adolescent computer use and alcohol use: What are the role of quantity and content of computer use?. Addict Behav. 2011; 36(5): 520-522.

27. Ito $M$, Baumer $S$, Bittanti $M$, et al. Hanging out, messing around and geeking out. Kids living and learning with new media. Massachusetts: The MIT Press; 2010. (Citado el 24 Marzo 2015). Disponible en https://mitpress.mit. e d u / s i tes/defa ult/files/titles/free download/9780262013369_Hanging_Out.pdf

28. Huang G, Unger J, Soto D, et al. Peer Influences: The impact of online and offline friendship networks on adolescent smoking and alcohol use. Journal of Adolescent Health. 2013; 54(2014): 508-514.

29. Bandura A. Social learning theory. New York: General Learning Press; 1977.

30. Bandura A. Social cognitive theory: an agentic perspective. Annual Review of Psychology. 2001; 52:1-26.

31. Moreno M, D’Angelo J, Kavincsky L, Kerr B, Zhang C, Eickoff, J. Emergence and predictors of alcohol reference displays on Facebook during the first year of college. Computers in Human Behavior; 2014; 30:87-94. doi: 10.1016/j.chb.2013.07.060.

\section{Correspondencia:}

Francisco Rafael Guzmán Facundo

Correo electrónico: francisco.guzmanf@uanl.mx

Fecha de Recepción: 05 de enero del 2016.

Fecha de aceptación: 15 de febrero del 2016. 